\title{
Properties of Symmetric Boolean functions
}

\author{
L. HAVIAROVÁ AND E. TOMAN
}

\begin{abstract}
In the present paper we consider symmetric Boolean functions with special property. We study properties of the maximal intervals of these functions. Later we show characteristics of corresponding interval graphs and simplified interval graphs. Specifically we prove, that these two graphs are isomorphic for symmetric Boolean function. Then we obtain the vertex degree of these graphs. We discuss also disjunctive normal forms.
\end{abstract}

Mathematics Subject Classification 2000: 05C80 (Random graphs), $60 \mathrm{C05}$ (Combinatorial probability), 68R10 (Graph theory), 06E30 (Boolean function)

Additional Key Words and Phrases: Symmetric Boolean function, interval graph, disjunctive normal form.

\section{INTRODUCTION}

A Boolean function can be represented by several types of graphs. Among them, the greatest attention has been devoted to the study of the graph $G(f)$ induced by the vertices of the $n$-cube, on which the Boolean function $f$ takes the value 1 . This geometric representation has been introduced by Jablonski in [1]. The concept of the interval graph and simplified interval graph of a Boolean function has been defined by Sapozhenko in [3].

In the present paper we study properties of symmetric Boolean functions. Boolean function is a symmetric Boolean function, if there exists a set $\left\{P_{1}, \ldots, P_{k}\right\}$, where $1 \leq k \leq n$ and $P_{i} \in\{0,1, \ldots, n\}$ while the function gains value 1 in the vertices which contain value 1 on $P_{i}$ positions. We denote level of Boolean cube $B^{n}$ as $P_{i}$ and symmetric Boolean function with levels $P_{1}, \ldots, P_{k}$ as $B_{P_{1}, \ldots, P_{k}}^{n}$.

The complexity of symmetric Boolean function was studied by Fagin, Klawe, Pippenger and Stockmeyer [6] and Denenberg, Gurevich and Shelah [7]. Their results were improved by Wegener [5]. Symmetric Boolean functions, expecially their cryptografic properties were studied by Canteaut and Videau [8].

Some results regarding disjunctive normal forms have already been obtained for almost all Boolean functions and were presented by Jablonski and Lupanov in [2]. In addition they described an algorithm for finding minimal d.n.f. for symmetric Boolean 
functions $B_{P_{i}, \ldots, P_{j}}^{n}$, where $i=j$. The principle of the algorithm is the numbering of the literals and cyclic movement of the indices. In this paper we extend this approach and prove results also for case $i \neq j$. Jablonski and Lupanov furthermore present asymptotic estimate for cases $i=\frac{n}{3}$ a $i=\frac{n}{4}$. It can be interesting to compare with our results.

\section{PRELIMINARIES AND NOTATION}

We use the standard notation from Boolean function theory. An $n$-ary Boolean function is a function $f:\{0,1\}^{n} \rightarrow\{0,1\}$.

We use an algebraic representation of Boolean functions - disjunctive normal form. A d.n.f. with minimal number of literals in this class is called the minimal d.n.f. of $f$ and the one with minimal length in this class is called the shortest d.n.f. of $f$.

We also use a geometric representation of Boolean functions. The Boolean $n$-cube is the graph $B^{n}$ with $2^{n}$ vertices $\tilde{\alpha}=\left(\alpha_{1}, \ldots, \alpha_{n}\right)$, where $\alpha_{i} \in\{0,1\}$, in which those pairs of vertices that differ in exactly one coordinate are joined with an edge. For an $n$-ary Boolean function $f$ let $N_{f}$ denote the subset $\{\tilde{\alpha} ; f(\tilde{\alpha})=1\}$ and $N_{f}^{-}$denote the subset $\{\tilde{\alpha} ; f(\tilde{\alpha})=0\}$ of all vertices $\tilde{\alpha}$. Notice that there is a one-to-one correspondence between the sets $N_{f}$ and Boolean functions $f$. The subgraph of the Boolean $n$-cube induced by the set $N_{f}$ is called the graph of $f$ and is denoted by $G(f)$.

The set of vertices $N_{i} \subseteq\{0,1\}^{n}$ corresponding to an elementary conjunction $K_{i}$ of rank $r$ is called the interval of rank $r$. Notice that to every elementary conjunction $K=x_{i_{1}}^{\alpha_{i_{1}}} \wedge \ldots \wedge x_{i_{r}}^{\alpha_{i_{r}}}$ there corresponds an interval of rank $r$ consisting of all vertices $\left(\beta_{1}, \ldots, \beta_{n}\right)$ of $B^{n}$ such that $\beta_{i_{j}}=\alpha_{i_{j}}$ for $j=1, \ldots, r$ and values of other vertex coordinates are arbitrary. In the present paper, we often work with intervals corresponding to elementary conjunctions.

In the geometric model, every interval of rank $r$ represents an $(n-r)$-dimensional subcube of $B^{n}$. Therefore we call the interval of rank $r$ also the $(n-r)$-dimensional interval. An interval $N$ is called the maximal interval of Boolean function $f$ if $N \subseteq N_{f}$ and there is no interval $N^{\prime} \subseteq N_{f}$ such that $N \subseteq N^{\prime}$. A d.n.f. which consists of all elementary conjunctions corresponding to maximal intervals is called the abbreviated d.n.f. and it is denoted by $D_{A}(f)$.

For an arbitrary Boolean function $f$ and each of its d.n.f.s $K_{1} \vee \ldots \vee K_{s}$, we have 
that

$$
N_{f}=\bigcup_{j=1}^{s} N_{j} .
$$

In other words, every d.n.f. of a Boolean function $f$ corresponds to a covering of $N_{f}$ by intervals $N_{1}, \ldots, N_{s}$ such that $N_{j} \subseteq N_{f}$. Conversely, every covering of $N_{f}$ by intervals $N_{1}, \ldots, N_{s}$ contained in $N_{f}$ corresponds to some d.n.f. of $f$. Using the geometric interpretation of d.n.f.s, we can express the irreducibility of d.n.f.. The d.n.f. $D$ of a Boolean function $f$ cannot be simplified if every interval $N_{j}$ of the covering corresponding to $D$ contains at least one vertex belonging to just this one interval of the covering. Such a d.n.f. is called an irredundant d.n.f..

Let $r_{j}$ denote the order of the interval $N_{j}$. Then the number of literals in d.n.f. is $r=\sum_{j=1}^{s} r_{j}$ and the construction of a minimal d.n.f. in the geometric model can be formulated as a problem of constructing a covering of $N_{f}$ by intervals $N_{j} \subseteq N_{f}$ with minimal $r$. On the other hand, the construction of a covering corresponding to the shortest d.n.f. requires to minimize the number of intervals in a covering of $N_{f}$.

The set of all conjuctions $K_{j}$ from $K_{1}, \ldots, K_{s}$ corresponding to intervals for which

$$
N_{j} \nsubseteq \bigcup_{\substack{i=1 \\ i \neq j}}^{s} N_{i}
$$

is called the core of d.n.f. $D=\bigvee_{j=1}^{s} K_{j}$ of a Boolean function $f$. It is denoted by $\gamma(D(f))$.

Now we can define the interval graph $\Gamma(f)$ as the graph associated with a Boolean function $f$ as follows: its vertices correspond to maximal intervals of $f$ and the vertices corresponding to intervals $N_{i}$ and $N_{j}$ are joined with an edge in $\Gamma(f)$ if $K_{i} \wedge K_{j}$ is nonempty.

Let us introduce the graph of Boolean function $f$ which we get from $\Gamma(f)$ by omitting all vertices corresponding to maximal intervals such that they belong to $\gamma\left(D_{A}(f)\right)$ or do not belong to any irreducible covering. Such a graph is called a symplified interval graph.

For more information about Boolean functions we suggest to see [11], as this paper is its extension. 


\section{PROPERTIES OF SYMMETRIC BOOLEAN FUNCTIONS}

In this section we study properties of symmetric Boolean function $B_{P_{i}, \ldots, P_{n-j}}^{n}, i+j<n$. We do not consider the trivial case $i+j=n$. We evaluate the number, dimension, radius and diameter of its maximal intervals. Then we discuss the characteristics of graphs $\Gamma$ and $\Gamma^{\prime}$ corresponding to $B_{P_{i}, \ldots, P_{n-j}}^{n}$. In the end we count the vertex degrees of interval and simplified interval graphs.

Let $\alpha$ be $(\underbrace{1, \ldots, 1}_{n})$ and $\beta$ be $(\underbrace{0, \ldots, 0}_{n})$.

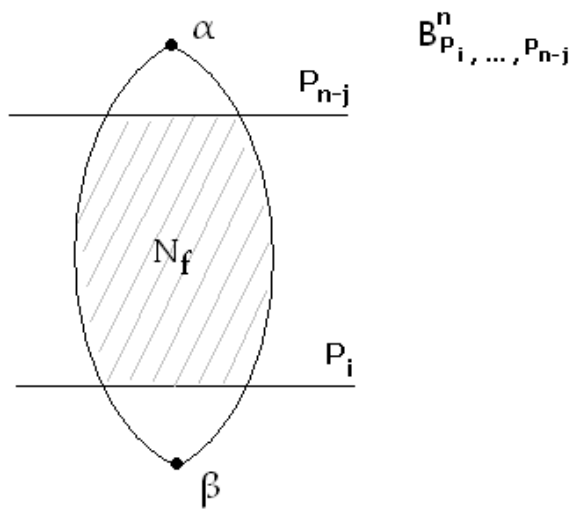

Fig. 1. Function $B_{P_{i}, \ldots, P_{n-j}}^{n}$

THEOREM 3.1. The dimension of maximal intervals of $B_{P_{i}, \ldots, P_{n-j}}^{n}$ is

$$
n-(i+j) \text {. }
$$

Proof. We know that $j$ levels from vertex $\alpha$ and $i$ levels from vertex $\beta$ belong to $N_{f}^{-}$. Therefore each vertex belonging to $N_{f}$ contains at least $i$ coordinates with value 1 and $j$ different coordinates with value 0 . The rest of the coordinates are arbitrary. It generates the intervals of dimension $n-(i+j)$. Inequality $i+j<n$ implies existence of such intervals.

COROLlary 3.2. The dimension of maximal intervals of $B_{P_{i}, \ldots, P_{n-i}}^{n}$ is

$$
n-2 i \text {. }
$$

The Theorem also implies, that all maximal intervals have the same dimension. Therefore all corresponding vertices in Interval graph have the same vertex degree. 
The graph $\Gamma\left(B_{P_{i}, \ldots, P_{n-i}}^{n}\right)$ is uniquely determined by the number of maximl interval and the vertex degree.

Let $\alpha^{\prime} \in N_{f}$ and $\beta^{\prime} \in N_{f}$ be the vertices of maximal interval containing at least $i$ coordinates with value 1 and at least $j$ coordinates with value 0 , respectively. Distance between $\alpha^{\prime}$ and $\beta^{\prime}$ is $n-(i+j)$. It is easy to see that $\alpha^{\prime}$ is situated on level $n-j$ and $\beta^{\prime}$ on level $i$.

THEOREM 3.3. The number of maximal intervals of $B_{P_{i}, \ldots, P_{n-j}}^{n}$ is

$$
\left(\begin{array}{c}
n-i \\
n-(i+j)
\end{array}\right) \cdot\left(\begin{array}{l}
n \\
i
\end{array}\right)
$$

PROOF. The number of edges between $\alpha^{\prime}$ and level $n-j+1$ is $n-j$. We already know that the dimension of all maximal intervals is $n-(i+j)$. Each maximal interval of dimension $k$ starting in $\alpha^{\prime}$ is uniquely determined by choosing $k$ edges between $\alpha^{\prime}$ and level $n-j+1$. Thus the number of different maximal intervals which contain vertex $\alpha^{\prime}$ and have the dimension $n-(i+j)$ is $\left(\begin{array}{c}n-j \\ n-(i+j)\end{array}\right)$. As there are $\left(\begin{array}{l}n \\ j\end{array}\right)$ possibilities of choosing $\alpha^{\prime}$ on level $n-j$, we get the result $\left(\begin{array}{c}n-j \\ n-(i+j)\end{array}\right) \cdot\left(\begin{array}{c}n \\ n-j\end{array}\right)$.

Result for vertex $\beta^{\prime}$ on level $i$ can be obtained analogicaly.

It holds that

$$
\left(\begin{array}{c}
n-i \\
n-(i+j)
\end{array}\right) \cdot\left(\begin{array}{l}
n \\
i
\end{array}\right)=\left(\begin{array}{c}
n-j \\
n-(i+j)
\end{array}\right) \cdot\left(\begin{array}{c}
n \\
n-j
\end{array}\right)
$$

which validate the Theorem.

COROLlaRY 3.4. The number of maximal intervals of $B_{P_{i}, \ldots, P_{n-i}}^{n}$ is

$$
\left(\begin{array}{c}
n-i \\
n-2 i
\end{array}\right) \cdot\left(\begin{array}{c}
n \\
i
\end{array}\right)
$$

THEOREM 3.5 .

$$
\left(\begin{array}{c}
n-[p n] \\
n-([p n]+[q n])
\end{array}\right) \cdot\left(\begin{array}{c}
n \\
{[p n]}
\end{array}\right) \sim \frac{1}{2 \pi n} \cdot \frac{1}{p^{[p n]+1 / 2}} \cdot \frac{1}{q^{[q n]+1 / 2}} \cdot \frac{1}{(1-p-q)^{n-[p n]-[q n]}},
$$

where $i=p . n, p<\frac{1}{2}, p, q \in Q,[x]$ stands for nearest integer to $x$.

PROOF. For the sake of simplicity let us assume $p n, q n$ are already their rounded versions in the remainder of this paper.

$$
\left(\begin{array}{c}
n-p n \\
n-(p n+q n)
\end{array}\right) \cdot\left(\begin{array}{c}
n \\
p n
\end{array}\right)=\frac{n !}{p n ! \cdot q n ! \cdot(n-(p n+q n)) !}
$$


Using Stirling formula we get

$$
\begin{gathered}
\frac{n !}{p n ! . q n ! .(n-(p n+q n)) !} \sim \\
\sim \frac{\left(\frac{n}{e}\right)^{n} \sqrt{2 \cdot \pi \cdot n}}{\left(\frac{q n}{e}\right)^{q n} \sqrt{2 \pi q n}\left(\frac{p n}{e}\right) p n \sqrt{2 \pi p n}\left(\frac{n-(p n+q n)}{e}\right)^{n-(p n+q n)} \sqrt{2 \pi(n-(p n+q n))}}= \\
=\frac{\sqrt{2 \pi n}}{\sqrt{2 \pi(n-(p n+q n))} \sqrt{2 \pi q n} \sqrt{2 \pi p n}} \cdot \frac{1}{p^{p n} q^{q n}} \cdot \frac{1}{(1-p-q)^{n-(p n+q n)}}= \\
=\frac{1}{2 \pi n} \cdot \frac{1}{p^{p n+1 / 2}} \cdot \frac{1}{q^{q n+1 / 2}} \cdot \frac{1}{(1-p-q)^{n-p n-q n}}
\end{gathered}
$$

THEOREM 3.6. For graphs $\Gamma$ and $\Gamma^{\prime}$ associated with the function $B_{P_{i}, \ldots, P_{n-j}}^{n}$ it holds that $\Gamma \cong \Gamma^{\prime}$.

PROOF. We divide the proof into two parts. First we show that none of maximal interval belongs to the core of $B_{P_{i}, \ldots, P_{n-j}}^{n}$. Then we proove that each maximal interval belongs to at least one irredundant d.n.f..

First part of the statement means that all vertices of each maximal interval are covered by another maximal interval of $B_{P_{i}, \ldots, P_{n-j}}^{n}$. Let $N_{I}$ be an arbitrary maximal interval with dimension $n-(i+j)$. $N_{I}$ contains $2^{n-(i+j)}$ vertices. Let $\delta \in N_{I}$ be an arbitrry vertex such that $\delta \in P_{k}, i \leq k \leq n-j$. Degree of vertex $\delta$ is $n$, with $n-(i+j)$ edges contained in $N_{I}$. Let us take a look at the remaining $i+j$ edges. According to the position of $N_{f}$ in $B^{n}, i$ and $j$ of them are incident with vertices on level $P_{k-1}$ and $P_{k+1}$ respectively, or vice versa. It implies that $i(j)$ edges are joined with level containing vertices from $N_{f}^{-}$. If we use one of these edges together with $n-(i+j)-1$ edges from $N_{I}$ we obtain maximal interval $N_{I}^{\prime}$ with dimension $n-(i+j)$ containing vertex $\delta$. Thus $N_{I}$ do not belong to the core.

Maximal interval does not belong to any irredundant d.n.f. iff all of its vertices are covered by core intervals [1]. This together with the first part completes the proof. 
THEOREM 3.7. The degree of each vertex of $\Gamma\left(B_{P_{i}, \ldots, P_{n-j}}^{n}\right)$ is

$$
\left.\sum_{m=0}^{n-(i+j)-1}(-1)^{m} \sum_{k=0}^{n-(i+j)-m}\left(\begin{array}{c}
n-(i+j) \\
m
\end{array}\right)\left(\begin{array}{c}
n-(i+j)-m \\
k
\end{array}\right)\left[\begin{array}{c}
n-m-k-i \\
n-m-k-(i+j)
\end{array}\right)\left(\begin{array}{c}
k+i \\
k
\end{array}\right)-1\right] .
$$

PROOF. Let $N_{I}$ be a maximal interval corresponding to $v_{N_{I}}$ in $\Gamma\left(B_{P_{i}, \ldots, P_{n-j}}^{n}\right)$. In order to count a vertex degree of $v_{N_{I}}$ we find out the number of maximal intervals which have nonempty intersection with $N_{I}$.

The process of evaluation consists of taking all intervals belonging to $N_{I}$, one at a time. Let the actual one be denoted as $N_{M}$. We count the maximal intervals different from $N_{I}$ such that $N_{M}$ is a subset of their intersection with $N_{I}$. Let $m$ be a summation variable adressing to dimension of $N_{M}$. According to Theorem 3.1. $m$ can gain values from 0 to $n-(i+j)-1$. To make sure that we count each maximal interval having nonempty intersection with $N_{I}$ only once, we use the inclusion-exclusion principle.

Figure 2 shows possible location of $N_{M}$. There are $n-(i+j)-m$ fixed coordinates in $N_{I}$, thus we have $\left(\begin{array}{c}n-(i+j) \\ m\end{array}\right)$ possibilities to choose $m$ arbitrary coordinates generating $N_{M}$. The other summation variable $k$ determines the position of interval $N_{M}, 0 \leq k \leq$ $n-(i+j)-m$. To do so, we choose $k$ coordinates with value 0 from $n-(i+j)-m$ fixed coordinates. It ensures that we go through all levels.

To sum it up there is $\left(\begin{array}{c}n-(i+j) \\ m\end{array}\right)\left(\begin{array}{c}n-(i+j)-m \\ k\end{array}\right)$ possibilities to choose intersection $N_{M}$.
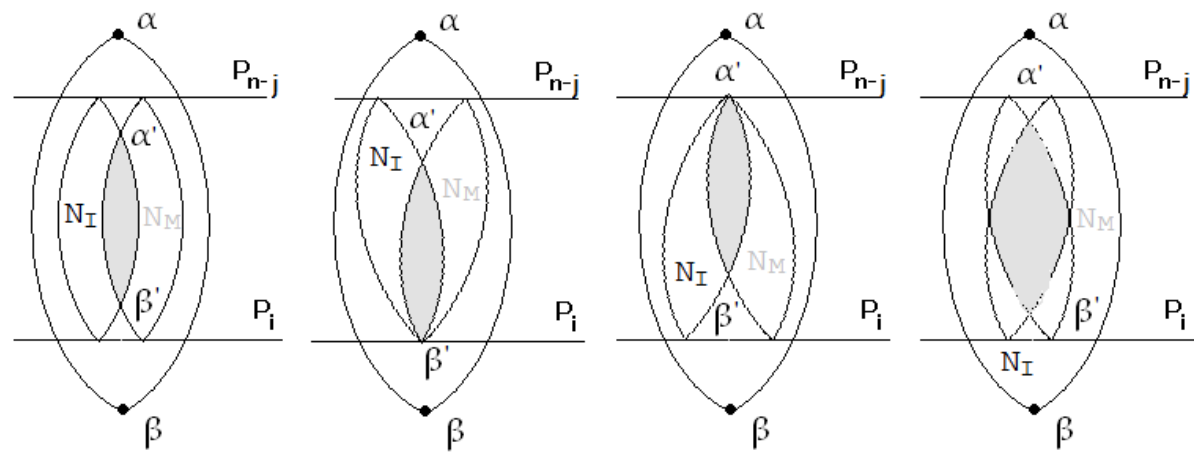

Fig. 2. Possible location of $N_{M}$ in $N_{I}$

Each feasible maximal intervals (different from $N_{I}$ and containing $N_{M}$ ) is uniquely defined by $n-(i+j)$ edges. $m$ of them generate $N_{M}$ so we need to choose aditional $n-(i+j)-m$ edges incident to $\alpha^{\prime} \in N_{M}$ or $\beta^{\prime} \in N_{M}$. 
We have to choose these edges in such way that the location of maximal intervals is between $i$ and $n-j$. Therefore we choose $k$ out of $k+i$ edges from $\alpha^{\prime} \in N_{M}$ towards $\alpha$. We have to pick other $n-(i+j)-m-k$ from the edges joined with $\beta^{\prime} \in N_{M}$ and leading to $\beta$. (There are $n-m-k-i$ such edges.)

The number $\left[\left(\begin{array}{c}n-m-k-i \\ n-m-k-(i+j)\end{array}\right)\left(\begin{array}{c}k+i \\ k\end{array}\right)-1\right]$ expresses the number of ways to choose other $n-(i+j)-m$ edges except the case when we choose interval $N_{I}$ itself.

Putting it all together we can see that the vertex degree of $v_{N_{I}}$ in $\Gamma\left(B_{P_{i}, \ldots, P_{n-j}}^{n}\right)$ is

$$
\sum_{m=0}^{n-(i+j)-1}(-1)^{m} \sum_{k=0}^{n-(i+j)-m}\left(\begin{array}{c}
n-(i+j) \\
m
\end{array}\right)\left(\begin{array}{c}
n-(i+j)-m \\
k
\end{array}\right)\left[\left(\begin{array}{c}
n-m-k-i \\
n-m-k-(i+j)
\end{array}\right)\left(\begin{array}{c}
k+i \\
k
\end{array}\right)-1\right] .
$$

COROLlary 3.8. The degree of each vertex of $\Gamma\left(B_{P_{i}, \ldots, P_{n-j}}^{n}\right)$ is

$$
\sum_{m=0}^{n-2 i-1}(-1)^{m} \sum_{k=0}^{n-2 i-m}\left(\begin{array}{c}
n-2 i \\
m
\end{array}\right)\left(\begin{array}{c}
n-2 i-m \\
k
\end{array}\right)\left[\left(\begin{array}{c}
n-m-k-i \\
n-m-k-2 i
\end{array}\right)\left(\begin{array}{c}
k+i \\
k
\end{array}\right)-1\right] .
$$

Vertex degree as a function of parameters $i$ and $n$ is computed and shown in the following Figure. 


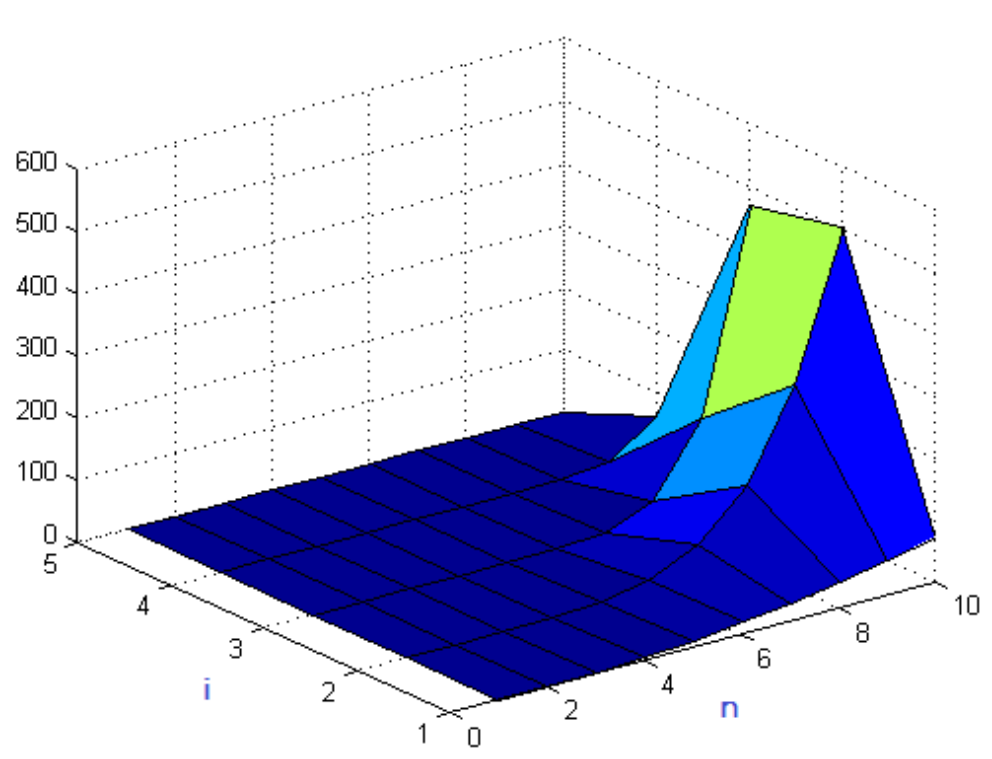

\begin{tabular}{|c|c|c|c|c|c|}
\hline $\mathrm{n}>\mathrm{i}$ & 1 & 2 & 3 & 4 & 5 \\
\hline 1 & 0 & 0 & 0 & 0 & 0 \\
\hline 2 & 0 & 0 & 0 & 0 & 0 \\
\hline 3 & 2 & 0 & 0 & 0 & 0 \\
\hline 4 & 6 & 0 & 0 & 0 & 0 \\
\hline 5 & 12 & 4 & 0 & 0 & 0 \\
\hline 6 & 20 & 18 & 0 & 0 & 0 \\
\hline 7 & 30 & 54 & 6 & 0 & 0 \\
\hline 8 & 42 & 130 & 36 & 0 & 0 \\
\hline 9 & 56 & 270 & 146 & 8 & 0 \\
\hline 10 & 72 & 504 & 470 & 60 & 0 \\
\hline
\end{tabular}

Fig. 3. Vertex degree, cases when $1<n<10,1<i<5$ 


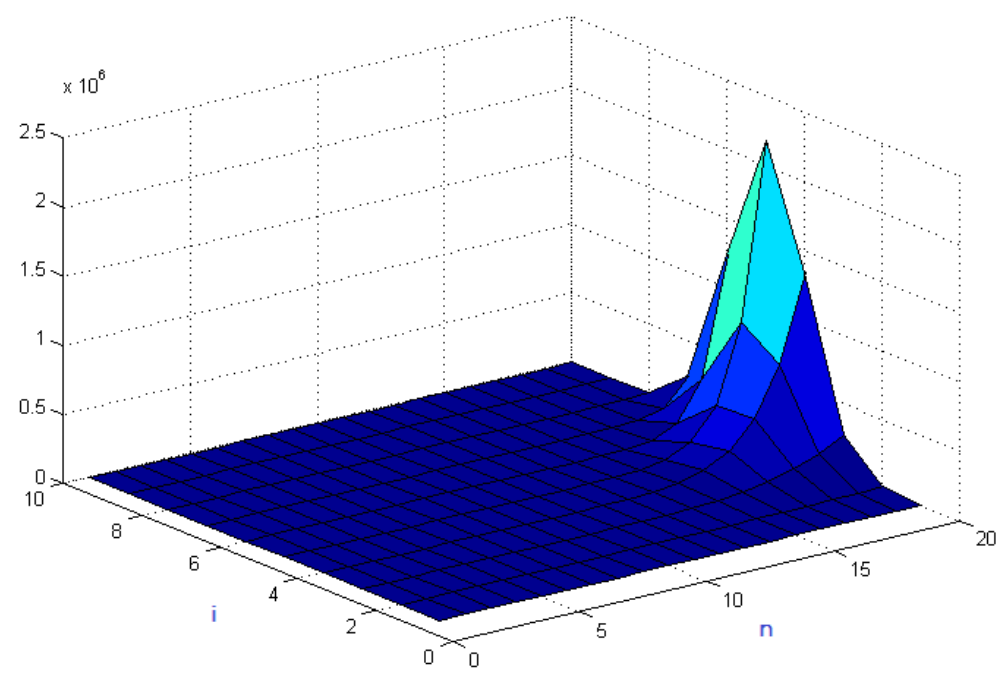

\begin{tabular}{|c|c|c|c|c|c|c|c|c|c|}
\hline $\mathrm{n}$ & 1 & 2 & 3 & 4 & 5 & 6 & 7 & 8 & 9 \\
\hline 1 & 0 & 0 & 0 & 0 & 0 & 0 & 0 & 0 & 0 \\
\hline 2 & 0 & 0 & 0 & 0 & 0 & 0 & 0 & 0 & 0 \\
\hline 3 & 2 & 0 & 0 & 0 & 0 & 0 & 0 & 0 & 0 \\
\hline 4 & 6 & 0 & 0 & 0 & 0 & 0 & 0 & 0 & 0 \\
\hline 5 & 12 & 4 & 0 & 0 & 0 & 0 & 0 & 0 & 0 \\
\hline 6 & 20 & 18 & 0 & 0 & 0 & 0 & 0 & 0 & 0 \\
\hline 7 & 30 & 54 & 6 & 0 & 0 & 0 & 0 & 0 & 0 \\
\hline 8 & 42 & 130 & 36 & 0 & 0 & 0 & 0 & 0 & 0 \\
\hline 9 & 56 & 270 & 146 & 8 & 0 & 0 & 0 & 0 & 0 \\
\hline 10 & 72 & 504 & 470 & 60 & 0 & 0 & 0 & 0 & 0 \\
\hline 11 & 90 & 868 & 1280 & 308 & 10 & 0 & 0 & 0 & 0 \\
\hline 12 & 110 & 1404 & 3066 & 1250 & 90 & 0 & 0 & 0 & 0 \\
\hline 13 & 132 & 2160 & 6636 & 4250 & 560 & 12 & 0 & 0 & 0 \\
\hline 14 & 156 & 3190 & 13236 & 12558 & 2750 & 126 & 0 & 0 & 0 \\
\hline 15 & 182 & 4554 & 24690 & 33110 & 11252 & 922 & 14 & 0 & 0 \\
\hline 16 & 210 & 6318 & 43560 & 79458 & 39732 & 5320 & 168 & 0 & 0 \\
\hline 17 & 240 & 8554 & 73326 & 176250 & 124222 & 25492 & 1414 & 16 & 0 \\
\hline 18 & 272 & 11340 & 118586 & 365750 & 350682 & 104958 & 9380 & 216 & 0 \\
\hline 19 & 306 & 14760 & 185276 & 716958 & 907752 & 380730 & 51562 & 2056 & 18 \\
\hline 20 & 342 & 18904 & 280910 & 1337960 & 2181256 & 1240398 & 242844 & 15420 & 270 \\
\hline
\end{tabular}

Fig. 4. Vertex degree, cases when $1<n<20,1<i<10$

In Figures 3-4 we illustrate obtained result. Red color represents $N_{f}^{-}$. 


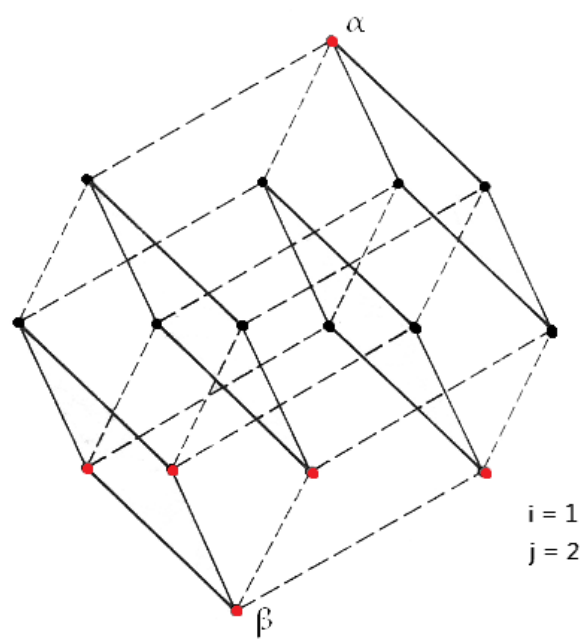

$\Gamma(f) \cong \Gamma^{\prime}(f)$

Fig. 5. Geometric representation of $B_{P_{2}, P_{3}}^{4}$

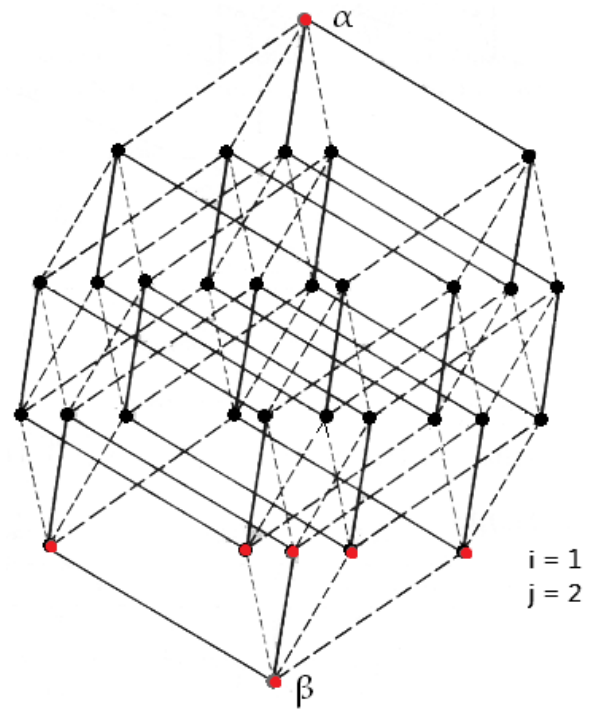

$\Gamma(f) \cong \Gamma^{\prime}(f)$

Fig. 6. Geometric representation of $B_{P_{2}, P_{3}, P_{4}}^{5}$ 


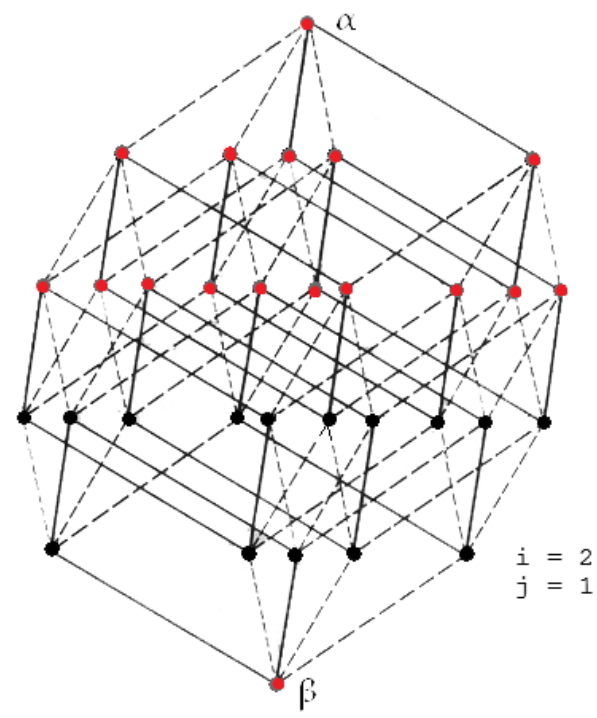

$\Gamma(f) \cong \Gamma^{\prime}(f)$

Fig. 7. Geometric representation of $B_{P_{1}, P_{2}}^{5}$

Definition 3.9. Let $G$ be a graph and let $\alpha, \beta \in G$. The distance from $\alpha$ to $\beta$ is the length of a shortest path from $\alpha$ to $\beta$. It is denoted by $d(\alpha, \beta)$.

Definition 3.10. The number $e(\alpha)=\max \{d(\alpha, \beta) \mid \beta \in G\}$ is called the excentricity of vertex $\alpha$.

Definition 3.11. The number $r(G)_{\alpha \in G}=\min \{e(\alpha)\}$ is called the radius of $G$.

Definition 3.12. The number $d(G)_{\alpha \in G}=\max \{e(\alpha)\}$ is called the diameter of $G$.

LEMMA 3.13. The excentricity of all vertices of an $n$-cube is equal to $n$.

PROOF. Distance between two vertices in an $n$-cube is given by the number of coordinates where the vertices differ. For each vertex in an $n$-cube there exists an "opposite" vertex which differs in all $n$ coordinates. It implies our statement.

Using this Lemma we directly obtain the following results.

THEOREM 3.14. The diameter of maximal intervals of $B_{P_{i}, \ldots, P_{n-j}}^{n}$ is $n-(i+j)$.

THEOREM 3.15. The radius of maximal intervals of $B_{P_{i}, \ldots, P_{n-j}}^{n}$ is $n-(i+j)$.

THEOREM 3.16. The diameter of subgraph of $B^{n}$ induced by $N_{f}$ of $B_{P_{i}, \ldots, P_{n-j}}^{n}$ is $\min (n, 2 n-2 \max (i, j))$. 
PROOF. We divide the proof into two cases.

First let us assume that $i \leq\lfloor n / 2\rfloor \wedge j \leq\lfloor n / 2\rfloor$. It implies that $N_{f}$ contains all vertices from level $n / 2$ (for $n$ even) or from levels $(n-1) / 2$ and $(n+1) / 2$ (for $n$ odd). For every vertex $\alpha$ from level $n / 2$ there exists vertex $\alpha^{\prime}$ with opposite coordinates from the same level (for $n$ even). Similarly for every vertex $\beta$ from level $(n-1) / 2$ there exists a vertex $\beta^{\prime}$ with opposite coordinates from level $(n+1) / 2$ (for $n$ odd). It is clear that in this case the diameter equals $n$.

Otherwise all vertices from $N_{f}$ contain at most $\lfloor n / 2\rfloor$ coordinates with value 0 (or $1)$. Let us refer to this value as $k$ and to its maximal appearance as $m$. Let us assume vertex $\delta$ which contains coordinate $k$ exactly $s \leq m$ times. The opposite vertex $\delta^{\prime} \in N_{f}$ can be found by negating as many coordinates as possible. It is possible to turn $s$ coordinates $k$ into $\bar{k}$ and $m$ coordinates $\bar{k}$ can be switched into $k$. Therefore excentricity of $\delta$ is given by $s+m$. As $s \leq m$ it implies that diameter equals $2 m$. It is clear that $m=n-\max (i, j)$ which completes the proof.

THEOREM 3.17. The radius of subgraph of $B^{n}$ induced by $N_{f}$ of $B_{P_{i}, \ldots, P_{n-j}}^{n}$ is $n-$ $|i-j|$.

PROOF. With the help of ideas from previous proof we obtain that vertices with the smallest excentricity are located on the level farthest from central levels. It can be the level $i$ if $i \leq j$ or $n-j$ if $i \geq j$. The vertex on level $n-j$ contains exactly $j$ zeros, vertex on level $i$ contains exactly $i$ ones. Let us look for the vertices with the maximal distance. Without loss of generality, let us choose $\alpha=\{\underbrace{1, \ldots, 1}_{n-j}, \underbrace{0, \ldots, 0}_{j}\}$ and $\beta=\{\underbrace{0, \ldots, 0}_{n-i} \underbrace{1, \ldots, 1}_{i}\}$. The length of the path between two vertices can be obtained as the number of identical coordinates subtracted from $n$. In the representation of vertices $\alpha$ and $\beta$ exists a section where coordinates of $\alpha$ and $\beta$ are equal. Its length is given by the difference between $i$ and $j$, in other words $|i-j|$. This facts implies that the diameter of subgraph is $n-|i-j|$.

Diameter and radius of graph induced by the set $N_{f}$ of Boolean function $B_{P_{2}, P_{3}, P_{4}}^{5}$ is illustrated in the following figure. 


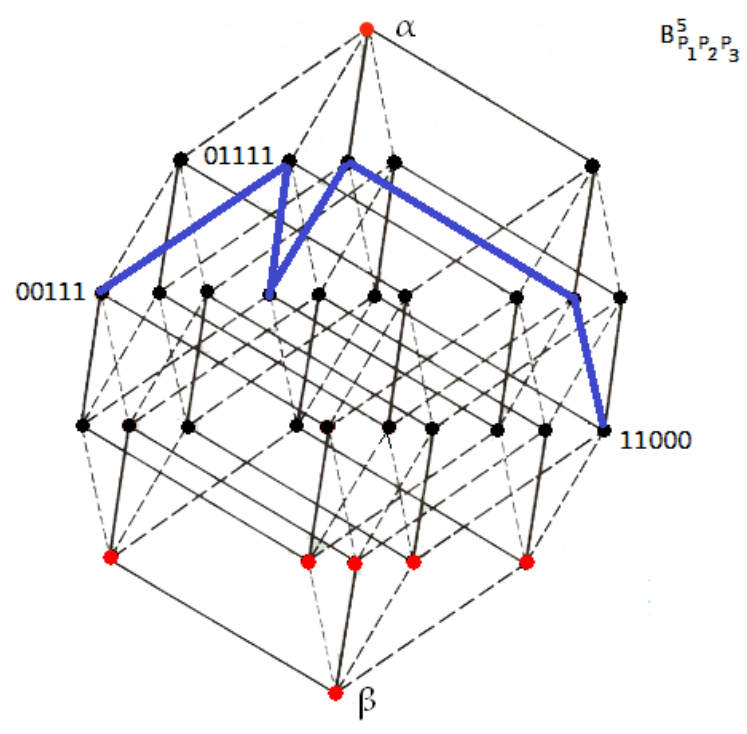

Fig. 8. An illustration of of radius and diameter

\section{DISJUNCTIVE NORMAL FORM}

In this section we take a look at disjunctive normal forms of $B_{P_{i}, \ldots, P_{n-j}}^{n}$.

REMARK 4.1. The fact that no maximal interval belongs to the core together with the definition of Quin's d.n.f gives us $D_{A}=D_{Q}$.

THEOREM 4.2. The number of conjunctions of an abbreviated d.n.f. of function $B_{P_{i}, \ldots, P_{n-j}}^{n}$ is

$$
\left(\begin{array}{c}
n-i \\
n-(i+j)
\end{array}\right) \cdot\left(\begin{array}{l}
n \\
i
\end{array}\right)
$$

PROOF. Straigthforward from Theorem 3.2.

According to the previous sections the following holds

$$
\left(\begin{array}{c}
n-p n \\
n-(p n+q n)
\end{array}\right) \cdot\left(\begin{array}{c}
n \\
p n
\end{array}\right) \sim \frac{1}{2 \pi n} \cdot \frac{1}{p^{p n+1 / 2}} \cdot \frac{1}{q^{q n+1 / 2}} \cdot \frac{1}{(1-p-q)^{n-p n-q n}},
$$

where $i=p . n, j=q . n, p<\frac{1}{2}, p \in Q$.

THEOREM 4.3. The number of conjunctions of a minimal d.n.f. of function 
$B_{P_{i}, \ldots, P_{n-j}}^{n}$ is

$$
\begin{aligned}
& \left(\begin{array}{l}
n \\
i
\end{array}\right), \quad \text { if } \quad i>j, \\
& \left(\begin{array}{l}
n \\
j
\end{array}\right), \quad \text { if } \quad i<j .
\end{aligned}
$$

PROOF. Let us divide the proof into two parts. In the first part we describe construction of minimal d.n.f. of the function $B_{P_{i}, \ldots, P_{n-j}}^{n}$ and verify its correctness for case $i<j$ and in the second part we repeat it for case $i>j$.

The dimension of maximal intervals corresponding to the conjunctions of minimal d.n.f. is $n-(i+j)$. It implies, that the number of literals in each conjunction is $i+j$. Maximal intervals are located between levels $i$ and $n-j$. As all vertices between these levels contain at least $i$ coordinates with value 1 and at least $j$ with value 0 , each conjunction contains $i$ non-negated variables and $j$ negated variables. Independently of the choice of arbitrary coordinates we never get the vertex from levels $0, \ldots, i-$ $1, n-j+1, \ldots, n$.

In the first part we consider the case $i<j$. There are $\left(\begin{array}{l}n \\ j\end{array}\right)$ different ways to choose $j$ negated variables out of $n$ variables. We get $\left(\begin{array}{l}n \\ j\end{array}\right)$ incomplete conjunctions, to which we add $i$ non-negated variables. They can be obtained by shifting the indices of negated variables. Let us perform this operation for $i$ variables with the biggest indices in a descending order. The index of each negated variables in the current conjunction is decreased to the closest suitable value. It means that there cannot be the same variable in negated and non-negated form at the same time in one conjunction. The shift operation is also cyclic. It is easy to see, that the set of non-negated and negated variables have an empty intersection. We join these two sets with operation AND. Using this construction we get $\left(\begin{array}{l}n \\ j\end{array}\right)$ different conjunctions.

Now we show the correctness of our construction. To achieve that, each vertex $\delta$ belonging to levels $i, \ldots, n-j$ is contained in at least one maximal interval corresponding to conjunction in our minimal d.n.f.. As there is at least one coordinate with value 1 and one with value 0 , there has to exist the pair of coordinates $(1,0)$ (indices are cyclic). In case that more such pairs exist, we always take one with the biggest index of variable corresponding to 0 . We omit this pair and repeat this operation $i$ times. As there are at least $i$ coordinates with each value, we can find $i$ such pairs, let us refer to these $0 \mathrm{~s}$ and $1 \mathrm{~s}$ as special $1 \mathrm{~s}$ and $0 \mathrm{~s}$. If we take those $i$ special zeros together with other $j-i$ randomly chosen ones and shift $i$ biggest ones of them as described above, we get the positions of $i$ special $1 \mathrm{~s}$. Hence 
$\delta$ is covered by the maximal interval with negated variables on places of chosen 0 s $(i$ special and $j-i$ random) and non-negated variables on places of special $1 \mathrm{~s}$.

It is easy to see that the number of maximal intervals cannot be lower than $\left(\begin{array}{l}n \\ j\end{array}\right)$ as at least one of the vertices on level $n-j$ would not be covered.

Second part of the proof can be obtained analogicaly.

It implies that the number of conjunctions of a minimal d.n.f. of function $B_{P_{i}, \ldots, P_{n-j}}^{n}$ is

$$
\begin{aligned}
& \left(\begin{array}{l}
n \\
i
\end{array}\right), \quad \text { if } \quad i>j, \\
& \left(\begin{array}{l}
n \\
j
\end{array}\right), \quad \text { if } \quad i<j .
\end{aligned}
$$

REMARK 4.4. As all maximal intervals have the same dimension (the number of literals in corresponding conjunctions), minimal d.n.f. of function $B_{P_{i}, \ldots, P_{n-j}}^{n}$ is also the shortest one.

Without loss of generality, let as asume that $i>j$.

THEOREM 4.5. It holds that

$$
\left(\begin{array}{c}
n \\
p n
\end{array}\right) \sim \frac{1}{2 \pi n} \cdot \frac{1}{p^{p n+1 / 2}} \cdot \frac{1}{(1-p)^{n-p n+1 / 2}},
$$

where $i=p . n, p<\frac{1}{2}, p \in Q$.

PROOF. Applying Stirling's formula we get

$$
\begin{gathered}
\left(\begin{array}{c}
n \\
p n
\end{array}\right) \sim \frac{\left(\frac{n}{e}\right)^{n} \sqrt{2 \pi n}}{\left(\frac{n-p n}{e}\right)^{n-p n} \cdot \sqrt{2 \pi(n-p n)} \cdot\left(\frac{p n}{e}\right)^{p n} \cdot \sqrt{2 \pi p n}}= \\
=\frac{1}{\sqrt{2 \pi p n} \sqrt{1-p}} \cdot \frac{1}{p^{p n}} \cdot \frac{1}{(1-p)^{n-p n}}=\frac{1}{2 \pi n} \cdot \frac{1}{p^{p n+1 / 2}} \cdot \frac{1}{(1-p)^{n-p n+1 / 2}} .
\end{gathered}
$$

These results can be further applied to evaluation of parameters of symmetric Boolean function $B_{P_{i_{1}}, \ldots, P_{i_{2}}, \ldots, P_{i_{k-1}}, \ldots, P_{i_{k}}}^{n}$ shown in the following Figure. 


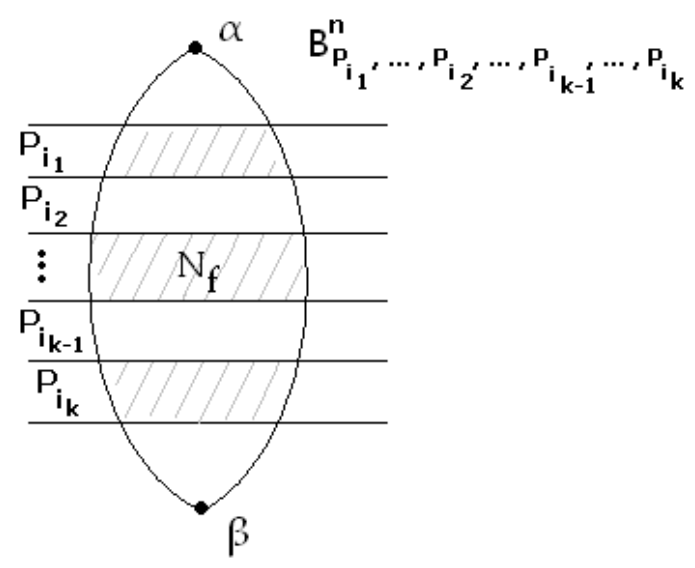

Fig. 9. Function $B_{P_{i_{1}}, \ldots, P_{i_{2}}, \ldots, P_{i_{k-1}}, \ldots, P_{i_{k}}}^{n}$

\section{EXAMPLES}

In this section we use obtained results to find asymptotic properties of maximal intervals, interval graphs and d.n.f.s of some chosen functions.

$$
\mathbf{i}=\mathbf{j}=\mathbf{n} / \mathbf{4}
$$

The number of maximal intervals, the length of abbreviated d.n.f., the number of vertices of $\Gamma$ :

$$
\begin{gathered}
\left(\frac{3 n / 4}{n / 2}\right) \cdot\left(\frac{n}{n / 4}\right)=\frac{n !}{\left(\frac{n}{2}\right) !\left(\frac{n}{4} !\right)^{2}} \sim \frac{\left(\frac{n}{e}\right)^{n} \sqrt{2 \cdot \pi \cdot n} \cdot e^{\frac{1}{12 \cdot n}}}{\left(\frac{n}{2 e}\right)^{\frac{n}{2}} \sqrt{\pi \cdot n} \cdot e^{\frac{1}{6 \cdot n}}\left(\frac{n}{4 e}\right)^{\frac{n}{2}} \frac{\pi \cdot n}{2} \cdot e^{\frac{2}{3 \cdot n}}}= \\
=\frac{2^{3 n / 2} \cdot \sqrt{2} \cdot 2}{\pi n} \cdot e^{1 / 12 n-2 / 6 n}=\frac{2^{\frac{3}{2}(n+1)}}{\pi n \cdot e^{1 / 4 n}}
\end{gathered}
$$

The dimension of maximal intervals: $n / 2$

The radius and diameter of maximal intervals: $\left\lceil\frac{n}{4}\right\rceil$

Full d.n.f.:

$$
\sum_{k=1 / 4}^{3 / 4}\left(\begin{array}{l}
n \\
k
\end{array}\right)=2^{n}-2 \cdot \sum_{k=0}^{1 / 4}\left(\begin{array}{l}
n \\
k
\end{array}\right)
$$

Using

$$
\sum_{k \leq \alpha . n}\left(\begin{array}{l}
n \\
k
\end{array}\right)=2^{n \cdot H(\alpha)-\frac{1}{2} \cdot \log n+O(1)}
$$


where $0<\alpha<\frac{1}{2}$ and $H(\alpha)=\alpha \cdot \log \frac{1}{\alpha}+(1-\alpha) \cdot \log \frac{1}{1-\alpha}$, from [9] for $\alpha=1 / 4$ we obtain

$$
\begin{gathered}
2^{n}-2 \cdot\left(2^{n .(1 / 4 \lg 4+3 / 4 \lg 4 / 3)-\frac{1}{2} \lg n / 4+O(1)}\right)= \\
=2^{n}-2^{1+\frac{n}{2}+\frac{3 n}{2}+3 n \text { over } 4 \lg 3+1-\frac{1}{2} \lg n+O(1)}= \\
=2^{n}-2^{2 n-\frac{3}{4} n \lg 3-\frac{1}{2} \lg n+O(1)}
\end{gathered}
$$

$$
\mathbf{i}=\mathbf{j}=\sqrt{\mathbf{n}} \log \mathbf{n}
$$

The number of maximal intervals, the length of abbreviated d.n.f., the number of vertices of $\Gamma$ :

$$
\begin{aligned}
& \left(\frac{n-\sqrt{n} \lg n}{n-2 \sqrt{n} \lg n}\right) \cdot\left(\frac{n}{\sqrt{n} \lg n}\right)=\frac{n !}{(n-2 \sqrt{n} \lg n) ![(\sqrt{n} \lg n) !]^{2}}= \\
& =\prod_{k=0}^{2 \sqrt{n} \lg n} \frac{(n-i)}{[(\sqrt{n} \lg n) !]^{2}}= \\
& =\frac{n(n-1)}{(\sqrt{n} \lg n)^{2}} \cdot \frac{(n-2)(n-3)}{(\sqrt{n} \lg n-1)^{2}} \cdot \frac{(n-2 \sqrt{n} \lg n+2)(n-2 \sqrt{n} \lg n+1)}{2} \sim \\
& \sim \frac{\left(\frac{n}{e}\right)^{n} \sqrt{2 \cdot \pi \cdot n} \cdot e^{\frac{1}{12 \cdot n}} \cdot e^{\frac{-1}{12(n-2 \sqrt{n} \lg n)} \cdot e^{\frac{-1}{6(\sqrt{n} \lg n)}}}}{\left(\frac{n-2 \sqrt{n} \lg n}{e}\right)^{n-2 \sqrt{n} \lg n} \cdot\left(\frac{\sqrt{n} \lg n}{e}\right)^{2 \sqrt{n} \lg n} \cdot \sqrt{2 \pi(n-2 \sqrt{n} \lg n)} \cdot(2 \pi \sqrt{n} \lg n)}= \\
& =\frac{\left(\frac{1}{1-\frac{2}{\sqrt{n}} \lg n}\right)^{n-2 \sqrt{n} \lg n+1 / 2}\left(\frac{\sqrt{n}}{\lg n}\right)^{2 \sqrt{n} \lg n}}{2 \pi \sqrt{n} \lg n}
\end{aligned}
$$

The dimension of maximal intervals: $n-2 \sqrt{n} \lg n$

The radius and diameter of maximal intervals: $\lceil n / 2-\sqrt{n} \lg n\rceil$

Full d.n.f.:

$$
\sum_{k=\sqrt{n} \lg n}^{n-\sqrt{n} \lg n}\left(\begin{array}{l}
n \\
k
\end{array}\right)=2^{n}-2 . \sum_{k=0}^{\sqrt{n} \lg n}\left(\begin{array}{l}
n \\
k
\end{array}\right)
$$

Using

$$
\sum_{k \leq \alpha . n}\left(\begin{array}{l}
n \\
k
\end{array}\right)=2^{n \cdot H(\alpha)-\frac{1}{2} \cdot \log n+O(1)}
$$


where $0<\alpha<\frac{1}{2}$ and $H(\alpha)=\alpha \cdot \log \frac{1}{\alpha}+(1-\alpha) \cdot \log \frac{1}{1-\alpha}, \mathrm{z}$ [9] for $\alpha=\frac{\lg n}{\sqrt{n}}$ we obtain

$$
\begin{gathered}
2^{n}-2 .\left(2^{n \cdot\left(\frac{\lg n}{\sqrt{n}} \lg \frac{1}{\frac{\lg n}{\sqrt{n}}}+\left(1-\frac{\lg n}{\sqrt{n}}\right) \lg \left(1-\frac{\lg n}{\sqrt{n}}\right)\right)-\frac{1}{2} \lg \sqrt{n} \lg n+O(1)}\right)= \\
=2^{n}-2^{1-\sqrt{n} \lg n \lg \lg n+1 / 2 \sqrt{n}(\lg n)^{2}-n \lg \left(1-\frac{\lg n}{\sqrt{n}}\right)+\sqrt{n} \lg n \lg \left(1-\frac{\lg n}{\sqrt{n}}\right)-1 / 4(\lg \sqrt{n})^{2}+O(1)}= \\
=2^{n}-2^{\sqrt{n} \lg n \lg \lg n+1 / 2 \sqrt{n}(\lg n)^{2}+\sqrt{n} \lg n-5 / 4(\lg n)^{2}+O(1)}
\end{gathered}
$$

We have chosen the case $i=\sqrt{n} \log n$ because the following holds [10]:

$$
\left|\bigcup_{t=n / 2-\sqrt{n} \log n}^{n / 2+\sqrt{n} \log n} B_{t}^{n}\right|=\left|B^{n}\right|=2^{n}
$$

\section{CONCLUSION}

In the present paper we have studied the properties of symmetric Boolean functions $B_{P_{i}, \ldots, P_{n-j}}^{n}, i+j<n$. We have evaluated the number, dimension, radius and diameter of its maximal intervals. We have also considered the characteristics of graphs $\Gamma$ and $\Gamma^{\prime}$ corresponding to $B_{P_{i}, \ldots, P_{n-j}}^{n}$. Then we have counted the vertex degrees of interval and simplified interval graphs. We have got some result regarding disjunctive normal forms. Finally we have presented examples to illustrate obtained result. 


\title{
REFERENCES
}

[1.] Jablonski S. V., Introduction into discrete mathematics, Moscow, Nauka, 1979 (in Russian).

[2.] Jablonski S. V. and Lupanov O. B., Discrete mathematics and Mathematical Problems of Cybernetics, Nauka, Moscow, 1974, (in Russian), pages 99148.

[3.] Sapozhenko A. A., Disjunctive Normal Forms, Moscow University Press, Moscow, 1975 (in Russian).

[4.] Toman E., Haviarova L., Properties of the interval graph of a Boolean function, Acta Mathematica Universitatis, 2013, Vol. LXXXII, 2 (2013), pages 191200.

[5.] Wegener I., The Complexity of Boolean Functions, New York: Wiley, 1987

[6.] Fagin R., Klawe M., Pippenger N. and Stockmeyer L., Bounded-depth, polynomial-size circuits for symmetric functions, Theoretical Computer Science, 1985, Vol. 36, pages 239250.

[7.] Denenberg L., Gurevich Y. and Shelah S., Definability by constant-depth polynomial-size circuits, Information and Control, 1986, Vol. 70(2/3), pages 216240.

[8.] Canteaut A. and Videau M., IEEE Transactions On Information Theory, Symmetric Boolean Functions, 2005.

[9.] Graham R., Knuth D., Patashnik O., Concrete Mathematics a Foundation for Computer Science, Addison-Wesley Publishing Company, 1989.

[10.] Nigmatulin R. G., The Complexity of Boolean Functions, Kazan, University Press, 1983.

[11.] Toman E., Haviarova L., The Number of Monotone and Self-Dual Boolean Functions, Journal of Applied Mathematics, Statistics and Informatics, 2014, Vol. 10, pages 93-111.

\author{
L. Haviarova and E. Toman \\ Comenius University \\ Faculty of Mathematics, Physics and Informatics \\ Department of Computer Science \\ Mlynska dolina, 84248 Bratislava \\ Slovak Republic
}

